\title{
Temperature and Electric Field Dependence of Conduction in Low-Density Polyethylene
}

\author{
JR Dennison and Jerilyn Brunson
}

\begin{abstract}
A traditional constant voltage conductivity test method was used to measure how the conductivity of highly insulating low-density polyethylene polymer films depends on applied electric field, repeated and prolonged electric field exposure, and sample temperature. The strength of the applied voltage was varied to determine the electric field dependence. At low electric field, the resistivity was measured from cryogenic temperatures to well above the glass transition temperature. Comparisons were made with a variety of models of the conduction mechanisms common in insulators, including transient polarization and diffusion and steady-state thermally activated hopping conductivity and variable range hopping conductivity, to determine which mechanisms were active for LDPE and to provide a better picture of its electrical behavior.
\end{abstract}

Index Terms - Conductivity, dielectric materials, electric field effects, polyethylene.

\section{INTRODUCTION}

$\mathrm{H}$ IGH resistivity insulating polymers are ubiquitous in use, easily tailored to address specific chemical requirements, and endless in their possible applications in new technology. The prevalence of these materials in the design of spacecraft and many other technology components places special emphasis on the electrical properties of the insulators, which are critical for anticipating and preventing potentially damaging charging phenomena [1], [2], [3]. Effects of local potential differences can range from any number of systematic errors, arcing to external plasmas, and in the extreme case, complete system failure due to a charge pulse generated by breakdown of the insulating material [1], [3], [4]. Long-term accumulation of charge can cause degradation of exterior surfaces, enhance contamination of the materials, and cause inaccuracies in measurement or information storage. The history of the sample becomes important as the behavior of the material is modified with further charging [5], [6]. Increasing the versatility and reliability of charge transport models and expanding the database of information for the electronic

Manuscript received February 8, 2008. This work was supported in part by the funding from the NASA Space Environments and Effects Program, the NASA James Webb Space Telescope Program through Goddard Space Flight Center, and the NASA Rocky Mountain Space Grant Consortium.

JR Dennison, is a professor with the Physics Department at Utah State University, Logan, UT 84321 USA (Phone: 435-797-2936; fax: 435-7972492; e-mail: JR.Dennison@usu.edu).

Jerilyn Brunson is graduate student with the Physics Department at Utah State University, Logan, UT 84321 USA (e-mail: Jeri.Brunson@aggiemail.usu.edu ). properties of insulating materials can assist designers in accommodating and mitigating these harmful effects [2].

Modeling and understanding the complex relationships between polymer components and their surroundings are fundamentally based on a detailed knowledge of how individual materials store and transport charge. The low charge mobility of insulators causes charge to accumulate where deposited, preventing uniform redistribution of charge and creating differential local electric fields and potentials. Improving the design models requires a better understanding of the physics of materials, particularly with respect to insulating polymers. The conductivity of the material is a key transport parameter in determining how deposited charge will redistribute throughout the system, how rapidly charge imbalances will dissipate, and what equilibrium potential will be established under given environmental conditions [7]. As the requirements for space missions extend to new regions of space and more stringent requirements are placed on spacecraft performance, it becomes necessary to better understand the underlying conduction mechanisms that determine the response of insulators to temperature, electric field dose rate and sample conditioning and history.

The objective of this experimental work was to perform detailed measurements of the resistivity of LDPE and to evaluate models for the temperature and electric field dependence of the conductivity. Low-density polyethylene (LDPE) was chosen as a good candidate for conductivity models testing. It is one of the most common and versatile polymers; high uniformity and high purity samples can easily be obtained for testing. Much is known about LDPE [8-12] and it is relatively well characterized. LDPE is also semicrystalline, which increases the likelihood that hopping conductivity is an appropriate model. The relatively high steady-state conductivity of LDPE at room temperature, on the order of $10^{-15}-10^{-18}(\Omega-\mathrm{cm})^{-1}$ [13], means it is measurable using the constant voltage resistivity test method.

\section{THEORETICAL BACKGROUND}

The charge transport properties of insulators are significantly different from those of conductors and semiconductors, and in general involve fundamentally different conduction mechanisms. For conduction by charge transport through a material, the conductivity (the ratio of current density to electric field, $\sigma=J / E$ ) is given as the product of the charge per carrier $q_{c}$, density of carriers $n$, and carrier mobility $\mu$, as $\sigma=q_{c} n \mu$. Both $n$ and $\mu$ can be functions of time through 
temporal changes in electric field, $E$, and temperature, $T$, or through temporal changes in the spatial distribution of charge carriers or the occupation of charge carrier states. Even in steady-state conditions both $n$ and $\mu$ can depend on the magnitudes of $E$ or $T$ and reflect the electronic structure of the material.

The primary conduction mechanism for conductors involves intraband excitation of electrons from filled extended states to empty extended states at only slightly higher energy within the same conduction band; this mechanism is not available to insulators since there are no empty states within the valence band (i.e., $n \rightarrow 0$ ).

Charge transport in semiconductors is primarily via thermally activated interband excitation of electrons from states in the valence band to states in the conduction band with activation energy equal to the band gap energy. However, this conduction mechanism is negligible in insulators at reasonable working temperatures (again, $n \rightarrow 0$ ); indeed, the distinction between semiconductors and insulators is that thermally activated transitions between extended states are highly improbable in insulators, because the band gap energy separating the states is much larger than the average thermal energy of the electrons. In well-ordered semiconductors these states are extended states, but can be localized for topologically (structurally) disordered states or chemically disordered (e.g., dopant or intrinsic defect) states. While this reduces the activation energy to as little as the separation between the conduction and valence band mobility edges, the gap is still much larger than the thermal energy.

Now consider five conduction mechanisms involving localized states that are active in insulators: the steady-state conductivity due to thermally activated hopping (TAH) $\sigma_{\text {TAH }}$, variable range hopping (VRH) $\sigma_{V R H}$, and photoexcitation or radiation induced conductivity (RIC) $\sigma_{\text {RIC }}$; as well as the transient conductivity due to diffusion and polarization. The most promising theories for explaining electrical behavior in insulating polymers are based on hopping conductivity models developed to understand charge transport in disordered semiconductors and amorphous solids [14]. These theories assume that electrons or holes are the primary charge carriers and that their motion through the material is governed by availability of localized states treated as potential wells or traps in a lattice. They are well tested for semiconductors, but remain largely unverified for insulators [14], in large part because it is difficult to appropriately define the nature of localized states used to determine carrier density and mobility in materials with such complex molecular structure and extreme disorder. Concentrations of impurity atoms or chains are difficult to quantify, the polymer chains do not lend themselves to the simplifications of a lattice construct, and polar groups attached to the chains have significant influence on carrier mobility. These polar groups can also contribute to an overall material polarization that influences the internal electric field felt by the carriers [15], [16].

The theory of thermal assisted hoping conductivity [14] $\sigma_{T A H}$, originally formulated for charge transport in ionic crystals [17], provides a model for the temperature and electric field dependence of hopping conductivity. For example, it can model the thermal excitation of charge carriers trapped in shallow wells (localized states) below the conduction band into extended states in the conduction band, which are subsequently retrapped in shallow localized states. Here the carrier mobility is proportional to a Boltzmann factor with the energy scale set by trap depth, $\Delta H$, times a Boltzmann factor with energy scale $\pm q_{e} E a$ gained (lost) by a charge carrier moving with (against) the electric field over a distance of the average trap separation $a$. The standard form of $\sigma_{T A H}(E, T)$ [14] can be expressed in terms of a conductivity scaling factor $\sigma_{\text {TAHo }}$, a temperature scaling factor $T_{A}$, and an electric field scaling factor $E_{A}$ as:

$\sigma_{T A H}(E, T)=\left\{\sigma_{T A H o}(T)\left(T_{A} / T\right) F_{A}\left(\beta_{A}\right) \exp \left(-T_{A} / T\right)\right\}$

where

$$
F_{A}\left(\beta_{A}\right) \equiv 1 / \beta_{A} \sinh \left(\beta_{A}\right)
$$

and the ratio of field energy to thermal energy is

$$
\begin{aligned}
\beta_{A} \equiv 4 E T_{A} / 3 E_{A} T=q_{C} E a / k_{B} T \\
\quad \text { with } \sigma_{T A H o}(T)=2 N(T) \vee q_{C}{ }^{2} a^{2} \\
T_{A} \equiv \Delta H / k_{B} \text { and } E_{A} \equiv 4 \Delta H / 3 q_{C} a
\end{aligned}
$$

$\sigma_{\text {TAHO }}(T)$ can be related to the frequency of hops, $v$, and can have a weak temperature dependence through energy density of charge carriers, $N(T)$.

At low electric fields $<10^{6} \mathrm{~V} / \mathrm{m},(1)$ is independent of $\mathrm{E}$; that is at low electric fields, $F_{A} \rightarrow 1$. In the range of $\sim 10^{6} \mathrm{~V} / \mathrm{m}$ to $10^{7} \mathrm{~V} / \mathrm{m}$ (1) is approximately linear in E. At still higher fields of $>10^{7} \mathrm{~V} / \mathrm{m}$, (1) becomes temperature independent and exhibits a $\exp \left(E^{1 / 4}\right)$ dependence. At largest electric fields near the electrostatic breakdown potential, the TAH conductivity model diverges. Numerous alternative models for the high electric field dependence have been developed [14] For example, application of a large electric field across the sample distorts the potential well and lowers the activation energy needed for the electron to hop the potential barrier $[14,18]$; this enhanced conductivity leads to the so-called PooleFrenkel factor [16] where $\beta_{P F}$ is the Poole-Frenkel coefficient that depends only on the charge of the carrier and the dielectric constant of the material, assuming a coulombic potential well $[19,20]$. Note that at low electric fields, $F_{P F} \rightarrow 1$. The Poole-Frenkel model is only a rough approximation, which has been extended in many ways [14]; of particular importance is application of the Onsanger treating steady-state diffusion of carriers between trapped states. 

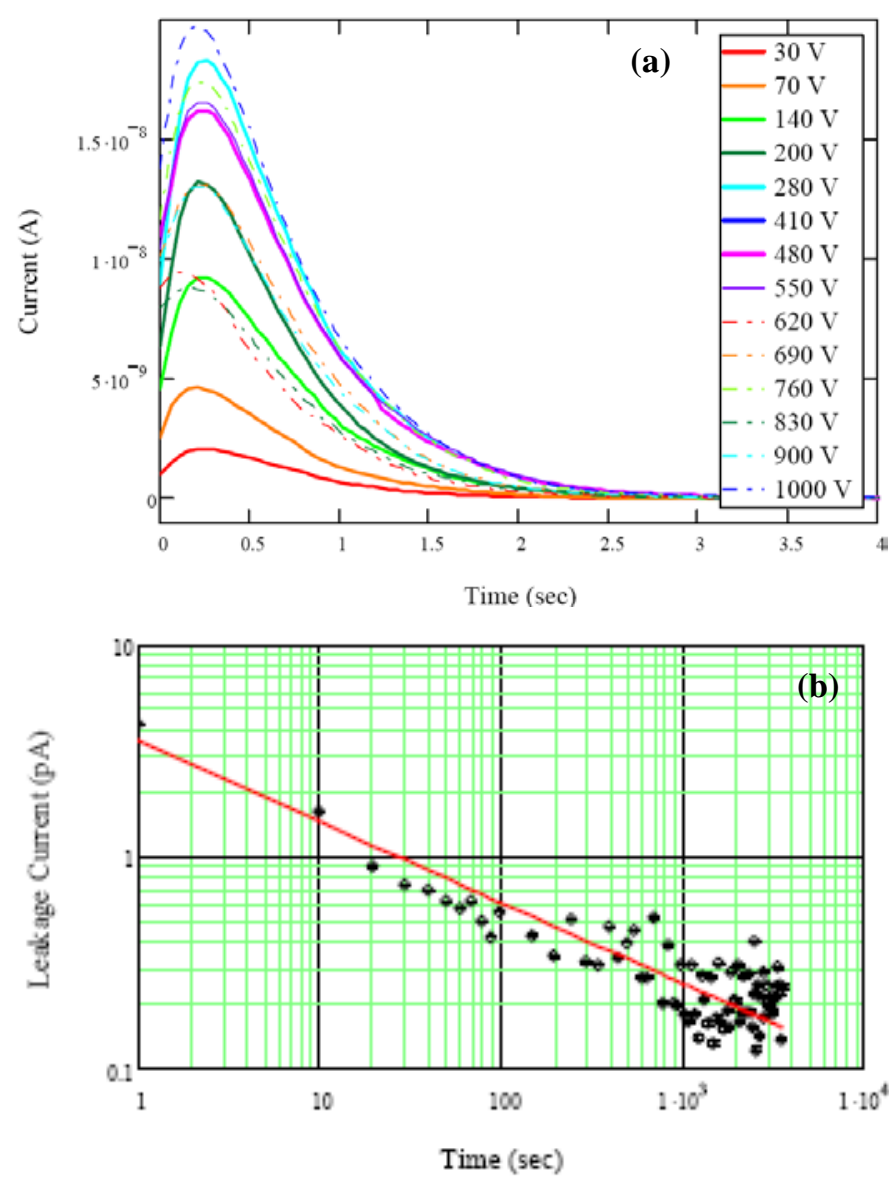

Fig. 1 lime dependence ot the sample current under applied voltage. (a) Initial current decay due to internal polarization for a series of 14 applied electric fields. (b) Leakage current through a $27 \mu \mathrm{m}$ LDPE sample at an applied voltage of $140( \pm 1) \mathrm{V}$ at $23( \pm 2){ }^{\circ} \mathrm{C}$ for one hour. The fit shown is a power law fit, $I(t)=I_{o} t^{-\alpha}$, with $I_{o}=3.5 \mathrm{pA}$ and a power law exponent $\alpha=0.38$.

The VRH conductivity mechanism developed by Mott and Davis [15], [18] models charge transport from one localized state to another, through thermally assisted quantum mechanical tunneling. It is often applied to a distribution of deeper trap states, such as localized impurity states within the band gap, where promotion to extended states is highly unlikely. Here the carrier mobility is proportional to the product of a Boltzmann factor with the energy scale set by the difference in trap depth of the localized states, $\Delta W$, and a tunneling probability, $\exp (2 R \alpha)$; this second term is proportional to the square of an exponent of the ratio of the well separation (or barrier width), $R$, to the localization length of the states (or wave function decay length), $\alpha^{-1}$. The possibility that an electron can tunnel to a more distant neighboring well with a larger energy difference leads to a more gradual decrease in conductivity, resulting in a $T^{-1 / 4}$ dependence in the exponent for 3-dimensional solids. This means that, even though the density of localized defect states in the gap that contribute to $\sigma_{V R H}$ is usually much less than for the localized states in the conduction band mobility edge that contribute to $\sigma_{T A H}$, there is the possibility that $\sigma_{V R H}$ can be dominant at low $\mathrm{T}$.
The standard form of Mott and Davis expression-as modified by Apsley and Hughes [21] [22] to include electric field dependence-can also be expressed in terms of a conductivity scaling factor $\sigma_{V R H o}$, a temperature scaling factor $T_{V}$, and an electric field scaling factor $E_{V}$ as:

$$
\sigma_{V R H}(E, T)=\left\{\sigma_{V R H o}(T)\left(T_{V} / T\right)^{1 / 4} F_{1}\left(\beta_{V}\right) \exp \left[\left(-T_{V} / T\right)^{1 / 4} F_{2}\left(\beta_{V}\right)\right]\right\}
$$

where

$\beta_{V} \equiv 4 E T_{V} / 3 E_{V} T=q_{C} E(2 \alpha)^{-1} / k_{B} T$

is again the ratio of field energy to thermal energy. $\sigma_{\text {TAHo }}$ has the same functional form as $\sigma_{T A H o}$ in (3), although $v, N(T)$, and the field distance, $(2 \alpha)^{-1}$, have different behavior. Note that both $F_{1}$ and $F_{2}$ are complex polynomial functions of $\beta_{V}$ and that at low electric fields, both $F_{1} \rightarrow 1$ and $F_{2} \rightarrow 1$ [21]. These functions are defined as

$$
\begin{aligned}
& F_{o}(\beta) \equiv \frac{(1+\beta / 2)}{(1+\beta)^{2}}+\left(1+\frac{3}{2} \beta\right) \\
& F_{1}(\beta) \equiv\left[2 / F_{o}(\beta)\right]^{1 / 4}
\end{aligned}
$$

and

$$
F_{2}(\beta) \equiv\left(\frac{-1}{2 \beta}\right) \cdot\left[1+\frac{F_{o}(\beta)}{F_{o}(\beta)-\frac{3}{2} \beta}\right] \cdot F_{1}(\beta) \cdot\left[\frac{\frac{3+\beta}{24 \cdot(1+\beta)^{3}}-\frac{1}{8}-\frac{\beta}{3}}{\frac{2+\beta}{6 \cdot(1+\beta)^{2}}+\frac{1}{3}+\frac{\beta}{2}}\right]
$$

Another steady-state conduction mechanism-called photoconductivity or radiation induced conductivity (RIC) involves excitation of carriers into extended states from either extended or localized states by external influences. This includes electron photoexcitation by light or by high energy radiation including electrons, ions and photons. A general model for RIC has the conductivity proportional to the $\Delta$ power of the dose rate (absorbed energy per unit mass and time)

$\sigma_{R I C}(\dot{D})=k(T) \dot{D}^{\Delta(T)}$

When $\sigma_{R I C}$ is not active, the remaining conditivity is referred to as the dark current conductivity. A recent investigation of RIC for LDPE is given elsewhere [23] and will not be considered further here.

A transient conduction mechanism, driven by spatial gradients in the charge distribution, is the diffusion conductivity given by

$\sigma_{\text {diff }}(t)=\left(q_{c} D_{o} / E\right) \partial p(t) / \partial z$

where $D_{o}$ is the carrier diffusion coefficient, $z$ is the depth into the sample, and $p(t)$ is the time-dependant spatial charge carrier density. For insulators diffusion often describes the 


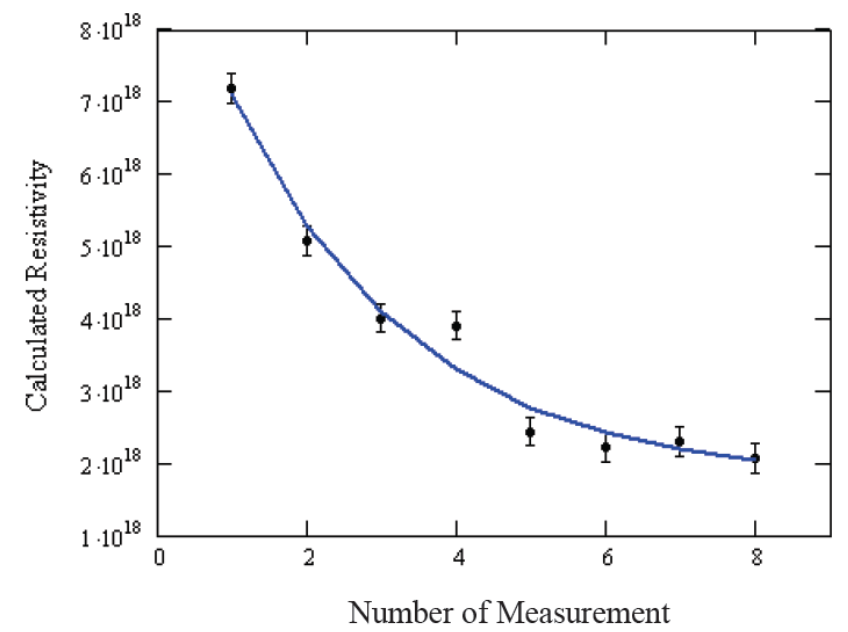

Fig. 2 Steady-state resistivity for a series of eight runs at $500( \pm 1) \mathrm{V}$ for $1 \mathrm{hr}$, separated by $1 \mathrm{hr}$ discharge times with the $27 \mu \mathrm{m}$ thick LDPE sample held at ground potential. The fit is an exponential decay (8).

spread of injected carriers into trapped states within the material. Space charge effects can be significant as traps are filled with injected charge and inhibit further motion of the carriers. Diffusion of particles to lattice sites often leads to a power law model of the time dependence of this conduction.

Finally, in insulators a displacement conduction mechanism results from the time dependant response of dielectric materials to an applied electric field. No net charge is transferred across the material; rather the transient dielectric current results primarily from the reorientation of molecular dipoles and the movement of ionic charge from one part of the sample to another in response to the applied field. In a simple relaxation time model of this charge displacement the current in parallel plate geometry for a constant applied voltage can be expressed as a time-dependant effective polarization conductivity

$\sigma_{P}(t)=\left[\varepsilon_{o}\left(\varepsilon_{r}^{\infty}-\varepsilon_{r}^{o}\right) / \tau_{P}\right] e^{-t / \tau_{P}}$

where $\varepsilon_{r}$ is the relative dielectric constant of the material and $\tau_{\mathrm{P}}$ is material polarization decay time for the polarization current to decay to $1 / \mathrm{e}$ of its initial value. For the constant voltage method, a macroscopic first-principles model has been developed [7] that contains both the initial current due to polarization and the long-time conduction current though the material. An example of transient polarization currents in lowdensity polyethylene (LDPE) is shown in Fig. 1. For LDPE, one hour is typically sufficient to ensure that all polarization currents have ceased to contribute to the measured current.

\section{EXPERIMENT AND PROCEDURE}

Samples of branched LDPE (Goodfellow, ASTM type I) of $27.4( \pm 0.2) \mu \mathrm{m}$ thickness had a density of $0.92 \mathrm{~g} / \mathrm{cm}^{3}$ [13] with an estimated crystallinity of 50\% [14] and a relative dielectric constant of 2.26 [13]. All samples were chemically cleaned with methanol prior to a bakeout at $65( \pm 1)^{\circ} \mathrm{C}$ under $\sim 10^{-3} \mathrm{~Pa}$ vacuum for $>24 \mathrm{hr}$ to eliminate absorbed water and volatile contaminants; samples conditioned in this manner had a measured outgassing rate of $<0.05 \%$ mass loss/day at the end of bakeout as determined with a modified ASTM 495 test procedure. Electrostatic breakdown field strength of conditioned samples was measured in a separate test chamber to be $2.9( \pm 0.3) 10^{8} \mathrm{~V} / \mathrm{m}$, using a modified ASTM D 3755 test procedure at room temperature under $<10^{-2} \mathrm{~Pa}$ vacuum with a voltage ramp rate of $20 \mathrm{~V}$ steps each sec. A similar test conducted in the constant voltage resistivity test chamber at a voltage ramp rate of $50 \mathrm{~V}$ steps each sec found an electrostatic breakdown field strength of $2.610^{8} \mathrm{~V} / \mathrm{m}$.

Conductivity of conditioned samples was measured in a constant voltage resistivity test chamber [24], using a modified ASTM D 257 test procedure [25] in which a thin film sample was placed between two electrodes, a voltage was applied across the sample, and the leakage current was measured. The measurements were made in a vacuum chamber maintained at $\sim 10^{-3} \mathrm{~Pa}$, with very good electrical shielding and low-noise cabling using guarded highly-polished OFHC $\mathrm{Cu}$ electrodes with an area of $2.0 \mathrm{~cm}^{2}$ and $\sim 0.2 \mathrm{MPa}$ clamping force per unit area. Preliminary measurements for different sample thicknesses and different electrode materials suggest that the condition of the sample/electrode interfaces do not appreciably affect the measured conduction current. Low temperatures were achieved using a l- $\mathrm{N}_{2}$ reservoir, while resistive heaters were used to reach high temperatures. Temperature was monitored to $\pm 0.5{ }^{\circ} \mathrm{C}$ with several Type $\mathrm{K}$ thermocouples attached to various parts of the apparatus.

Voltage, $V$, was applied with dc power supplies up to $\sim 6500( \pm 1) \mathrm{V}$ at a voltage ramp rate of $50 \mathrm{~V}$ steps each sec. Current, $I$, was measured at $0.1 \mathrm{sec}$ intervals for the initial current decay curves shown in Fig. 1a, and at increasing intervals up to $10 \mathrm{sec}$ at longer times as shown in Fig. 1b. Dissipation of diffusion and polarization currents dictated that the relevant time scale of the measurements were much longer durations than the 1 min suggested in ASTM D 257 [25]. The resistivity for samples of area $A$ and thickness $d$ was calculated using the thin film capacitor approximation, $\rho=V A / I d$ [5], [7]. Under certain conditions measurements for highly resistive materials involved extremely small steadystate leakage currents that approached the instrumentation limit of the electrometer (Keithley Model 616, 3 fA limit in lowest range). The minimum current base noise level alone corresponds to a resistivity of $\rho_{\text {res }}=7( \pm 2) \cdot 10^{18} \Omega$-cm at the lowest applied voltage; higher applied voltages allow measurements of resistivities up to $10^{20} \Omega$-cm.

Separate samples were used in for each experiment, except as noted, because it has been shown in previous work [20] that charging history and repeated applied voltages have an effect on the resistivity of the sample. (Refer to Fig. 2.)

\section{A. Electric Field Dependence}

To determine the electric field dependence of the conductivity at room temperature, a sample was placed under an applied voltage for one hour and then allowed a thirty minute recovery period with no applied voltage before the next applied voltage run. A series of 15 runs at increasing 


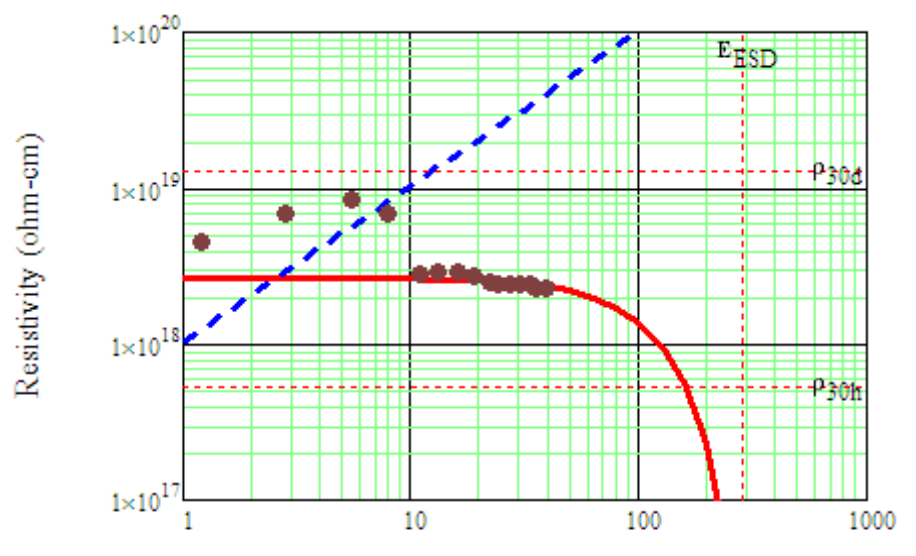

Electric Field (MV/m)

Fig. 3 Dark current steady-state resistivity as a function of electric field at room temperature. Dotted blue line indicates the instrumental resolution. Solid red line is hopping conductivity model fit., based on the sum of (1) and (3).

applied voltages from $30 \mathrm{~V}$ to $1000 \mathrm{~V}$, corresponding $1 \cdot \mathrm{Mv} / \mathrm{m}$ to $36 \mathrm{MV} / \mathrm{m}$ or $0.4 \%$ to $13 \%$ of the measured breakdown voltage, were measured at room temperature, $23( \pm 2){ }^{\circ} \mathrm{C}$. The measured leakage current as a function of time for each run for the first $4 \mathrm{sec}$ is shown in Figure 1a. Figure 1b shows a typical current under $140( \pm 1) \mathrm{V}$ at $23( \pm 2){ }^{\circ} \mathrm{C}$ measured up to $1 \mathrm{hr}$. For each experimental run, the steady-state resistivity was calculated based on the average current and voltage over the last $\sim 10$ min of the run. The resistivity as a function of $E$ at room temperature is shown in Fig. 3.

Additional studies like the ones above have investigated the effects of sample electric field conditioning and space charge accumulation by varying the order and duration of applied voltages and the discharge time between sequential runs. One such example is shown in Fig. 2 where an LDPE sample was subjected to a series of eight runs at $500( \pm 1) \mathrm{V}$ for $1 \mathrm{hr}$, separated by $1 \mathrm{hr}$ discharge times with the sample held at ground potential.

\section{B. Temperature Dependence}

Measurements of the temperature dependence of resistivity of LDPE were done in two stages. The chamber and sample were cooled to $-40{ }^{\circ} \mathrm{C}$ using liquid nitrogen and, once equilibrium was reached, placed under an applied voltage of $140( \pm 1) \mathrm{V}$. The lower bound of the experimental temperature range was chosen to avoid possible onset of anomalous behavior due to structural changes near the lower working temperature of $-60{ }^{\circ} \mathrm{C}[13]$ and the glass transition temperature near $-80{ }^{\circ} \mathrm{C}$ [14]. Changes in resistivity due to such a structural phase transition are beyond the scope of this research.

Once temperature equilibrium was reached, the chamber and samples were then allowed to return to room temperature without the aid of internal or external heating, and the leakage current was monitored throughout the warming period. A typical warming period without intervening heating lasted $\sim 20$ $\mathrm{hr}$, corresponding to an average warming rate of $\sim 3^{\circ} \mathrm{C} / \mathrm{hr}$. A second temperature scan was then measured as the sample was gradually heated from $20{ }^{\circ} \mathrm{C}$ to $55{ }^{\circ} \mathrm{C}$ and the current

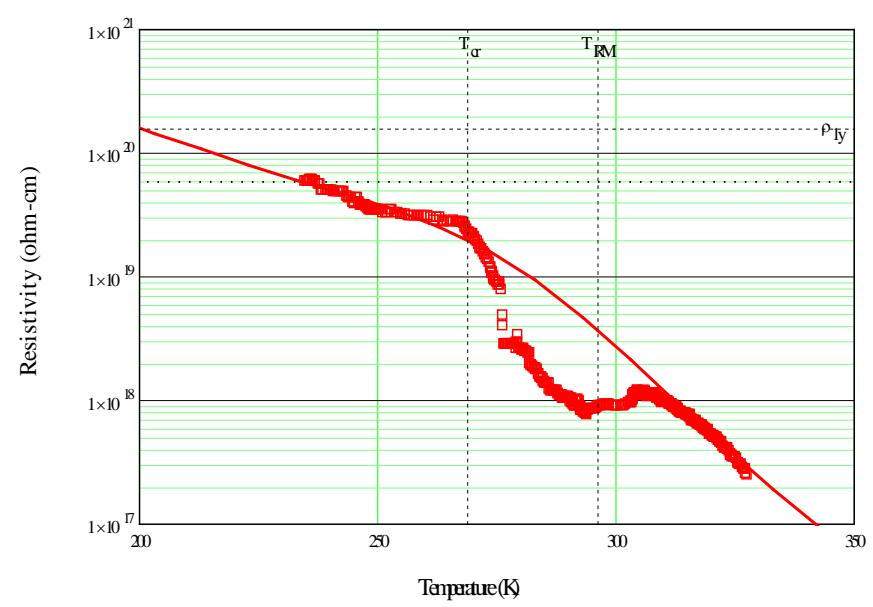

Fig. 4 Dark current steady-state resistivity as a function of temperature under an applied voltage of $140( \pm 1) \mathrm{V}$. Measurements of the temperature scans of of a $27 \mu \mathrm{m}$ LDPE sample were done in two stages for temperatures above and below $\sim 293 \mathrm{~K}$. Vertical dashed lines indicate the critical temperature, $\mathrm{T}_{\mathrm{cr}}=268 \mathrm{~K}$, and room temperature, $\mathrm{T}_{\mathrm{RM}}=293 \mathrm{~K}$. Lower horizontal dashed line indicates the instrumental resolution.

monitored. The leakage current through the sample was found to be sensitive to variations in heating rate, which may have caused a difference in the resistivity for the warmest parts of the cooling run where the heating rate was quite slow. Problems with temperature stability and more rapid heating in the lower temperature region of the heating scan resulted in less reliable data, most likely due to the sample resistivity not reaching equilibrium.

The measured leakage current over the course of the entire temperature range is shown in Fig. 4. Additional measurements over the full temperature range and extending to lower temperatures are in progress.

\section{Results AND ANALYSIS}

The initial time dependence of the current displayed in Fig. 1a shows a rapid exponential decrease, typical of a polarization conduction mechanism described by (7). The initial rise in current before $0.5 \mathrm{sec}$ is attributed to the response time of the voltage supply. All runs exhibited a similar exponential decay with an average polarization decay time $\tau_{\mathrm{P}}=0.56( \pm 0.04)$ sec over the first $\sim 5 \mathrm{sec}$, independent of the applied electric field up to $\sim 36 \mathrm{MV} / \mathrm{m}$. Such a rapid polarization decay time is consistent with the fact that polyethylene is composed of a non-polar monomer.

The long term time response shown in Figure 1b exhibits a power law decay typical of a diffusion current described by (7). The fit shown is a power law fit, $I(t)=I_{o} t^{-\alpha}$, with $I_{0}=3.5$ pA and a power law exponent $\alpha=0.38$. The value of $\alpha$ is consistent with the process of charge injection forming trapped space charge, with an exponent of 1 for full spacecharge limited decay and $<1$ when there is significant trapping, regardless of the specific charge injection mechanism involved [14]. Adamec and Calderwood observed a similar power law behavior with $\alpha<0.4$ at times $>10 \mathrm{sec}$; however, they argue that the conduction mechanism is due neither to polarization nor to space charge effects [9]. By 
contrast, references [11] and [12] present strong arguments for the presence of space charge trapping mechanisms in LDPE.

The decrease in resistivity by a factor of $\sim 5$ with continued application of voltage shown in Fig. 2 confirms that the space charge limit is achieved only after $\sim 5 \mathrm{hr}$ at $500 \mathrm{~V}$. Data in Fig 2 can be fit with a decaying exponential of the form

$\rho_{s c}(t)=\left(\rho_{s c}^{o}-\rho_{s c}^{\infty}\right) e^{-t / \tau_{S C}}+\rho_{s c}^{\infty}$

where $\rho_{\mathrm{SC}}{ }^{0}$ and $\rho_{\mathrm{SC}}{ }^{\infty}$ are the zero space charge and full space charge, resistivities, with values of $9.2 \cdot 10^{18} \Omega$-cm and $1.9 \cdot 10^{18}$ $\Omega$-cm respectively. Assuming that trapped charge does not appreciably dissipate during the $1 \mathrm{hr}$ relaxation periods between successive $1 \mathrm{hr}$ voltage applications, the space charge decay constant is $4.1 \mathrm{hr}$. The electric field dependence of $\alpha$ and $\tau_{S C}$ are currently being investigated to better characterize the specific charge injection mechanism(s) involved.

The steady-state resistivity calculated for electric field and temperature dependant runs are shown in Figures 3 and 4 respectively. While the measured time and voltage duration dependence of resistivity discussed above, suggest that the samples may not have reached complete steady-state equilibrium, the results have nevertheless been fit by the sum of (1) and (3) for the electric field and temperature dependant TAH and VRH steady-state conductivity mechanisms, respectively. The best overall fit, shown in Figures 3 and 4, has fitting parameters of $\sigma_{\text {TAHO }}=8.0 \cdot 10^{-8}(\Omega-\mathrm{cm})^{-1}, T_{A}=$ $8.9 \cdot 10^{3} \mathrm{~K}, E_{A}=9.5 \cdot 10^{8} \mathrm{~V} / \mathrm{m}, \sigma_{V R H o}=1.0 \cdot 10^{-10}(\Omega-\mathrm{cm})^{-1}, T_{V}=$ $1.0 \cdot 10^{8} \mathrm{~K}$, and $E_{V}=6.9 \cdot 10^{13} \mathrm{~V} / \mathrm{m}$. Based on (2b) for $\sigma_{T A H}$ and similar equations for $\sigma_{V R H}$, these fitting parameters correspond to an average well depth $\Delta H=0.76 \mathrm{eV}$, an average well spacing $a=1.1 \mathrm{~nm}$ and an average trap site separation $\alpha^{-1}=2.9 \mathrm{~nm}$. These values are in surprisingly good agreement with activation energy of $(0.78 \mathrm{eV}$ [11]; $0.87 \mathrm{eV}$ [8]; $0.80 \mathrm{eV}$ to $0.83 \mathrm{eV}$ [12]; and $0.6 \mathrm{eV}$ to $1.1 \mathrm{eV}$ [10]) and a trap site separation (2.8 $\mathrm{nm}$ [8] and $2.0 \mathrm{eV}$ at $303 \mathrm{~K}$ [10]) from previous studies of LDPE conduction.

The electric field dependence of LDPE resistivity shown in Fig. 3 is in reasonable agreement with the TAH theory in Eq. (1). For these data, at room temperature, the VRH contribution is expected to be negligible. Note that there are large uncertainties for the measured points below $10 \mathrm{MV} / \mathrm{m}$, since these resistivities are larger than the approximate instrumental resolution, as indicated by the dashed blue line in Fig. 3. Resistivities from $10 \mathrm{MV} / \mathrm{m}$ to $40 \mathrm{MV} / \mathrm{m}$ show a small decrease with increasing electric field as is predicted by the TAH model. Also note that, as expected, the predicted resistivity curve diverges at the electrostatic breakdown field strength measured independently as $\sim 310^{8} \mathrm{~V} / \mathrm{m}$ (see Section III).

Data acquired in two separate temperature scans from $233.4( \pm 0.5) \mathrm{K}$ to $293.4( \pm 0.5) \mathrm{K}$ and $293.4( \pm 0.5) \mathrm{K}$ to $328.4( \pm 0.5) \mathrm{K}$ were concatenated and are shown together in a semi-log plot in Fig. 4. The temperature dependence of LDPE resistivity also shows reasonable agreement with TAH and VRH theory. The resistivity does show two distinct regions below and above a critical temperature at $T_{c r}=268 \mathrm{~K}$ where the TAH and VRH conductivities are equal, with an abrupt decrease in the slope of the resistivity temperature profile at lower temperatures, corresponding to the theoretical onset of variable-range hopping where it is energetically favorable for the electron to hop to lower energy states beyond the nearest neighbor states [18]. At high temperatures the data follow a more rapid change with temperature predicted by the TAH model of (1). At low temperatures the data follow exhibit a more gradual temperature change, consistent with the VRH model of (3).

Additional room temperature measurements at higher field strengths, temperature scans at higher field strengths, and better temperature data near room temperature are required to better validate the model. Further investigations are also required to ensure that a variable temperature rate is not a significant factor in the temperature dependent behavior of the resistivity.

\section{CONCLUSION}

In general, the results presented in this study support the proposed models for charge transport in LDPE. The rapid exponential decay of the initial current, with a decay constant of $<1 \mathrm{sec}$ that is independent of electric field is consistent with a fast polarization decay time resulting from the largely nonpolar atomic structure of LDPE. The power law dependence of the transient current at longer times with a power law exponent of somewhat less than one are consistent with a diffusion of charges to trapped sites on a time scale of hours. This is corroborated by an observed decrease in resistivity for prolonged application of electric field, attributed to a process of charge injection into trapped states reaching the full space charge limit only after many hours.

The steady-state resistivity of LDPE is found to be consistent with a general model comprised of both thermally activated hopping and variable range hopping. Two distinct regimes of temperature dependence are observed; below 268 $\mathrm{K}$ the resistivity has a $\mathrm{T}^{-1 / 4}$ dependence characteristic of VRH and above $268 \mathrm{~K}$ the resistivity has a $\mathrm{T}^{-1}$ dependence characteristic of TAH. The electric field dependence is also consistent with the model, although the range of electric field measurements is too limited to provide a strong test of the model. The values of the six fitting parameters for the steadystate model produce estimates of the average well depth and well spacings that are consistent with the results of previous studies.

However, one must be very careful in over-interpreting the identification of specific mechanisms for conduction in LDPE, as many different physical models produce similar trends in the temporal, temperature and electric field dependence of conductivity [14]. More research is needed to determine which mechanisms that can induce similar E-field behavior and result in similar temperature dependence are responsible for conduction in the LDPE polymer. 


\section{REFERENCES}

[1] D. Hastings, H. Garrett, Spacecraft-Environment Interactions, New York, NY: Cambridge Press, 1996.

[2] J.R. Dennison, C.D. Thomson, J. Kite, V. Zavyalov, and Jodie Corbridge, "Materials Characterization at Utah State University: Facilities and Knowledgebase of Electronic Properties of Materials Applicable to Spacecraft Charging," Proceedings of the $8^{\text {th }}$ Spacecraft Charging Technology Conference, (NASA Marshall Space Flight Center, Huntsville, AL, October 2003).

[3] R.D Leach and M.B. Alexander, "Failures and anomalies attributed to spacecraft charging," NASA Reference Publication 1375, NASA Marshall Space Flight Center, August 1995.

[4] A.R. Frederickson and C.E. Benson, "Improved Testing Procedures For Spacecraft Discharge", Proceedings of the $7^{\text {th }}$ Spacecraft Charging Technology Conference, (ESTEC, Noordwijk, The Netherlands, April 2001).

[5] J.R. Dennison, A.R. Frederickson, and Prasanna Swaminathan, "Charge Storage, Conductivity, and Charge Profiles of Insulators As Related to Spacecraft Charging," Proceedings of the $8^{\text {th }}$ Spacecraft Charging Technology Conference, (NASA Marshall Space Flight Center, Huntsville, AL, October 2003).

[6] A.R. Frederickson and J.R. Dennison, "Measurement of Conductivity and Charge Storage in Insulators Related to Spacecraft Charging," IEEE Transaction on Nuclear Science, 50(6), 2284-2291 (December 2003).

[7] J.R. Dennison, Prasanna Swaminathan, Randy Jost, and Jerilyn Brunson, Nelson W. Green, and A. Robb Frederickson, "Proposed Modifications To Engineering Design Guidelines Related To Resistivity Measurements And Spacecraft Charging," Proceedings of the $9^{\text {th }}$ Spacecraft Charging Technology Conference, (Epochal Tsukuba, Tsukuba, Japab, April 4-8, 2005).

[8] R. Nath and M. M. Perlman, "Steady-state bulk trap-modulated hopping conduction in doped linear low-density polyethylene,” J. Appl. Phys. 65, 4854-4858, 1989.

[9] V. Adamec and J. H. Calderwood, "On the determination of electrical conductivity in polyethylene,” J. Phys. D: Appl. Phys., 14, 1487-1494, 1981.

[10] L. Boudou and J. Guastavino, "Influence of temperature on low-density polyethylene films through conduction measurement,” J. Phys. D: Appl Phys. 35, 1555-1561, 2002.

[11] J. N Marat-Mendes, R. M. Neagu and E. R. Neagu, "Electrical conduction and space charge trapping in highly insulating materials," J. Phys. D: Appl. Phys, 37, 343-347, 2004.

[12] G.C. Montanari, G. Mazzanti, F. Palmieri, A. Motori, G. Perego and S. Serra, "Space-charge trapping and conduction in LDPE, HDPE and XLPE,” J. Phys. D: Appl. Phys., 34, 2902-2911, 2001.

[13] "Material Information - Polyethylene Low Density LDPE," .Goodfellow, Devon, PA, January 20, 2006.

[14] H.J. Wintle, "Conduction Processes in Polymers," in Engineering Dielectrics - Volume IIA: Electrical Properties of Solid Insulating Materials: Molecular Structure and Electrical Behavior, American Society for Testing and Materials, R. Bartnikas, Editors, (American Society for Testing and Materials, Philadelphia, PA 19103, 1983).

[15] N.F. Mott, Phil. Mag. 19, 835 (1969).

[16] H.N. Poole, Philosophical Magazine, 34, 195-204, 1917.

[17] A. T. Amos and R. J. Crispin, J. Chem. Phys., 63, 1890-1899, 1975.

[18] N.F. Mott and E.A. Davis, Electronic Processes in Non-Crystalline Materials, $2^{\text {nd }}$ Ed. (Oxford University Press, Oxford, 1979).

[19] H. Böttger and V.V. Bryskin, Hopping Conduction in Solids. (Akademie-Verlag, Berlin, 1985).

[20] Jerilyn Brunson and J.R. Dennison, "E-Field Conditioning and Charging Memory in Low Density Polyethylene," American Physical Society Four Corner Section Meeting, Utah State University, Logan, UT, October 6-7, 2006.

[21] N Apsley and P H Hughes, "Temperature- and field-dependence of hopping conduction in disordered systems," Phil. Mag. 30 963-972, 1974.

[22] N Apsley and P H Hughes, "Temperature- and field-dependence of hopping conduction in disordered systems, II," Phil. Mag. 31 13271339, 1975.

[23] JR Dennison, Jodie Gillespie, Joshua Hodges, RC Hoffmann, J Abbott, Alan W. Hunt and Randy Spalding, "Radiation Induced Conductivity of Highly-Insulating Spacecraft Materials," Proceedings of the $10^{\text {th }}$ Spacecraft Charging and Technology Conference, (Biarritz, France, June 18-21, 2007).
[24] J.R. Dennison, Prasanna Swaminathan, Randy Jost, Jerilyn Brunson, Nelson Green, and A. Robb Frederickson, "Improved Methods and Analysis for Resistivity Measurements Related to Spacecraft Charging," IEEE Transaction on Plasma Science, 34(5) October 2006, 2191-2203.

[25] ASTM D 257-99, "Standard Test Methods for DC Resistance or Conductance of Insulating Materials" (American Society for Testing and Materials, 100 Barr Harbor Drive, West Conshohocken, PA 19428, 1999).

J. R. Dennison received a BS in Physics from Appalachian State University in Boone, NC in 1980, and MS and PhD degrees in Physics from Virginia Tech in Blacksburg, VA in 1983 and 1985, respectively. He was a Research associate at the University of Missouri-Columbia before moving to Utah State University in Logan, UT in 1988. He is presently a Professor of Physics at USU where he leads the Materials Physics Group. Dr. Dennison has worked in the area of electron scattering for his entire career and has focused on the electron emission and resistivity of materials related to spacecraft charging for the last fifteen years.

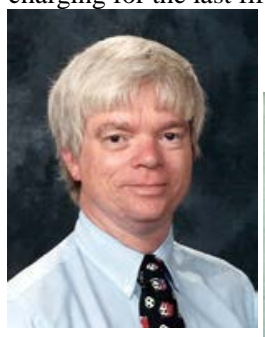

Jerilyn Brunson received her BS in Physics in 2003 from Utah State University in Logan, UT. She is currently a $\mathrm{PhD}$ student in Physics at USU. She has worked in the Materials Research group for six years, specializing in studies of the resistivity of highly insulating polymer materials. 